\title{
Aborted donor hepatectomy in living donor liver transplantation: lessons learned
}

\author{
K Kutluturk, (D) S Akbulut, A A Baskiran, (D) F Gonultas, (D) A Dirican, (D) B Isık, (D) S Yilmaz \\ Liver Transplant Institute, Inonu University Faculty of Medicine, Turkey
}

Corresponding author, email: akbulutsami@gmail.com

Background: Aborted donor hepatectomy (ADH) during any stage of living donor hepatectomy (LDH) is a rare event. We describe our experience and discuss the lessons from these events.

Methods: From September 2005 to January 2019, 77 of 2031 (3.79\%) LDH were aborted at various stages of surgical procedure due to donor or recipient related reasons. Demographic and clinical data of aborted donor candidates and the clinical course of their potential recipients were analysed.

Results: LDH of 77 donor candidates was aborted due to donor $(n=53)$ or recipient $(n=24)$ related reasons. The most common donor related reason was the quality of liver parenchyma $(n=31)$. The most common recipient related reason was haemodynamic instability $(n=11)$. Twenty-three recipients underwent either living donor liver transplantation (LDLT) $(n=21)$ or deceased donor liver transplantation (DDLT) $(n=2)$ at a median of 6 days following ADH. In one aborted due to a donor reason and two aborted for recipient reasons, LDLT was performed using the same donor candidates. Thirty-six recipients had no liver transplantation (LT) and died a median of 17.5 days following ADH.

Conclusions: We believe that $\mathrm{ADH}$ will decrease with experience and meticulous preoperative clinical and radiological evaluations. Abandoning the donor hepatectomy is always a valid option at any stage of the surgery when the unexpected is encountered.

Keywords: living donor liver transplantation; living donor hepatectomy, aborted donor hepatectomy; no-go donor hepatectomy, beginning syndrome

\section{Background}

Since the first successful liver transplantation (LT) was performed by Starzl and colleagues in 1967, LT has become the gold standard therapeutic modality for acute liver failure, chronic end stage liver disease and liver tumors. ${ }^{1}$ Living donor liver transplantation (LDLT) constitutes the majority of LT in many Asian and Middle East countries including Turkey where organ donation is insufficient mostly due to religious reasons, while deceased donor liver transplantation (DDLT) constitutes the majority of LT in western countries where cadaveric organ donation is the dominant. ${ }^{2,3}$ According to the a recent study, deceased and living donation rates for per million people in Spain, South Korea and Turkey were 43.6/8.0, 11.4/42.4 7.0/45.1, respectively. ${ }^{3}$ According to 2013 data of the Turkish Ministry of Health, 468 patients died on the waiting list and $77.8 \%$ of the patients who had brain death could not be used as cadaver donors because their relatives did not approve. ${ }^{3}$ Therefore, LDLT is the main solution in countries such as Turkey where cadaveric organ donation does not meet the needs of the patients on the waiting list.

Safety of living liver donor (LLD) in LDLT is the most important issue and preoperative detailed analysis of the donor and recipient candidates is mandatory to reduce the postoperative donor and recipient morbidity and mortality. ${ }^{4}$ Despite detailed preoperative evaluation, the living donor hepatectomy $(\mathrm{LDH})$ can be terminated due to various reasons at any stage of the surgical procedure. LDH can be aborted because of unpredictable or inevitable reasons related to donors or recipients and this is called aborted donor hepatectomy (ADH) or "no-go" donor hepatectomy. ${ }^{5-9}$ This condition can be avoided by the experience of the LDLT centre and use of advanced imaging techniques. However, unpredictable intraoperative findings related to donor or recipient can result in $\mathrm{ADH}$ even if all preoperative work-up is normal. Transplant centres at the beginning of the learning curve for performing LDLT have higher rates of $\mathrm{ADH}$, a condition we refer to as the "beginner syndrome". ADH or no-go donor hepatectomy was highlighted for the first time by Pomfret and colleagues. ${ }^{10-11}$ After then, a limited number of studies have been published about ADH..$^{5-11}$ In the present study we aimed to evaluate the results and implications of ADHs at our institute.

\section{Material and methods}

\section{Study cohort}

From September 2005 to January 2019, 2 031 LLD candidates were taken to the operating theatre for $\mathrm{LDH}$. LDH was successfully completed in 1954 LLD candidates. The surgical procedures of the remaining 77 LLD were terminated at various stages of the surgery due to donor or recipient related reasons (Figure 1). LLD candidates whose LDH procedure was terminated after abdominal incision were included in this study, while LLD candidates who refused the surgery in the operating theatre due to 


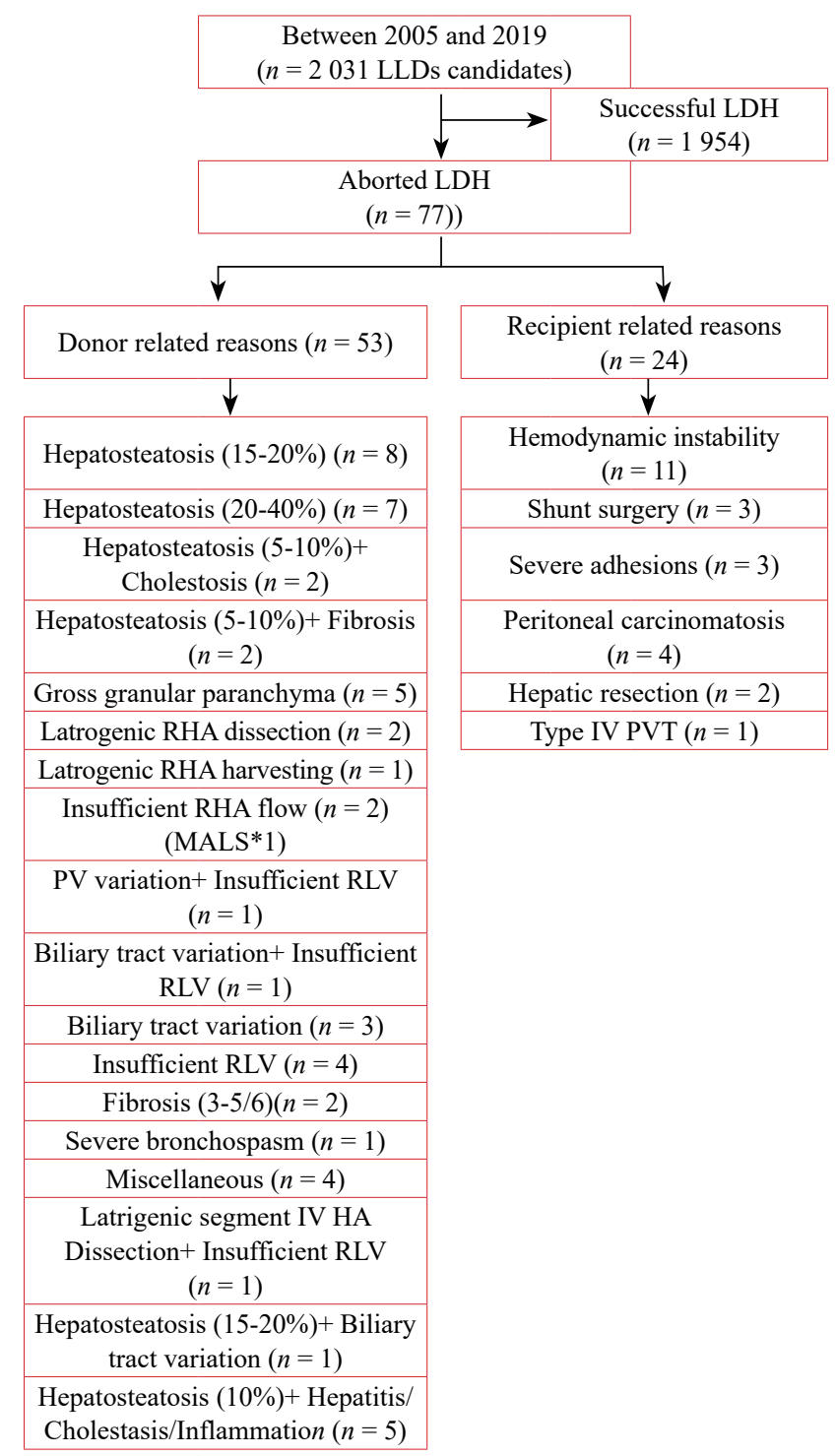

Figure 1: Classification of aborted donor hepatectomies according to underlying causes

psychosocial reasons were excluded. The surgeons and the anaesthesiologists together took the decision to terminate the surgical procedure in case of respiratory or haemodynamic instability during recipient and donor surgery. On the other hand, the surgical team decided to terminate the surgery for LLD candidates who had abnormalities in the bilio-vascular structures or adverse histopathological findings of the donor's liver. Similarly, it was a surgical team decision to terminate a recipient procedure due to technical issues such as advanced tumours or dense abdominal adhesions.

Patients' medical records were retrospectively reviewed and the following data was extracted and analysed: donor age (years), donor gender (male, female), donor BMI $\left(\mathrm{kg} / \mathrm{m}^{2}\right)$, reason for termination, the stage of termination of the operation and clinical course of recipients. ADHs were divided into two main categories: donor and recipient related reasons and results are presented based on these categories.

\section{Donor selection criteria}

Our country allows first to fourth degree relatives to become LLD candidates in accordance with the regulations of the ministry of health. Any LLD candidate that is not a relative of the recipient must be approved by the local ethics committee. The local ethics committee is composed of the governor of the state, the chief of police and the local administrator of the ministry of health. The ethics committee rule out any conflict of interest between the donor and the recipient before surgery. In our institution, all LDLTs performed from non-next of kin donors are approved by the local ethics committee.

The LLDs evaluation algorithm used in Inonu University LiverTransplant Institute has been previously described. ${ }^{12}$ The LLD candidates between 18 and 60 years with normal renal and liver function tests, negative viral serology panel except total hepatitis B core antibody positivity, and with a matched or compatible blood group type were further considered for radiologic evaluation. Magnetic resonance imaging (MRI) and magnetic resonance cholangiopancreatography (MRCP), initially used selectively, have now for the last two years become standard at our institute. Contrast enhanced multidetector computerised tomography (MDCT) was used to evaluate the vascular anatomy, graft volume, remnant liver volume and hepatosteatosis. Biliary tract anatomy was evaluated with both preoperative MRCP and conventional intraoperative cholangiography obtained before hilar dissection.

For the first ten years of our experience, LLD candidates were directly eliminated when $\geq 10 \%$ hepatosteatosis was detected in the MDCT. However, when there was no other available LLD candidate and there was a $5-10 \%$ hepatosteatosis in the MDCT or MRI, liver biopsy was performed mostly by using mini upper midline laparotomy or laparoscopy. The optimal LLDs candidates and recipients characteristics required for the LDLT procedure are as follows: graft to recipient weight ratio (GRWR) $\geq 0.8 \%$, graft weight/standard liver volume ratio $\geq 40 \%$ and the remnant liver volume $\geq 30 \% \cdot{ }^{13-15}$ However, if the recipient does not have any other LLD candidate or if portal venous pressure can be modulated, GRWR can be reduced to $0.7 \% .{ }^{15}$ If the LLDs are younger than 35 years and hepatosteatosis was not detected in histopathological examination, remnant liver volume can be reduced to $28 \% .{ }^{15}$ For the last three years of our experience, we have started to consider the donor selection criteria that are proposed by Lee and colleagues from Asian Medical Centre in addition to the standard donor evaluation criteria. These criteria consist of the age, fatty change (macrosteatosis) and remnant liver volume. ${ }^{16}$

\section{Literature review}

The second objective of this study was to review articles published in the literature on ADH. A literature search was conducted on PubMed, Medline, Google Scholar, and Google databases using the following terms: LT, LDLT, LLD, LDH, no-go LDH, and ADH. Data is presented in tabular form to give general and specific context to the current report.

\section{Results}

\section{General assessment}

The relationship of the donors to the recipients are as follows: unrelated $(n=6)$ and related $(n=71)$. Seventyseven of $2031(3.79 \%) \mathrm{LDH}$ procedure was terminated due to either recipient $(n=24)$ or donor $(n=53)$ related reasons in patients whose age ranged between 18 and 57 (median: 36 , mean \pm SD: $37.3 \pm 9.9$ ) years. The BMI of the LLD candidates ranged between 18 and $35.4 \mathrm{~kg} / \mathrm{m}^{2}$ 


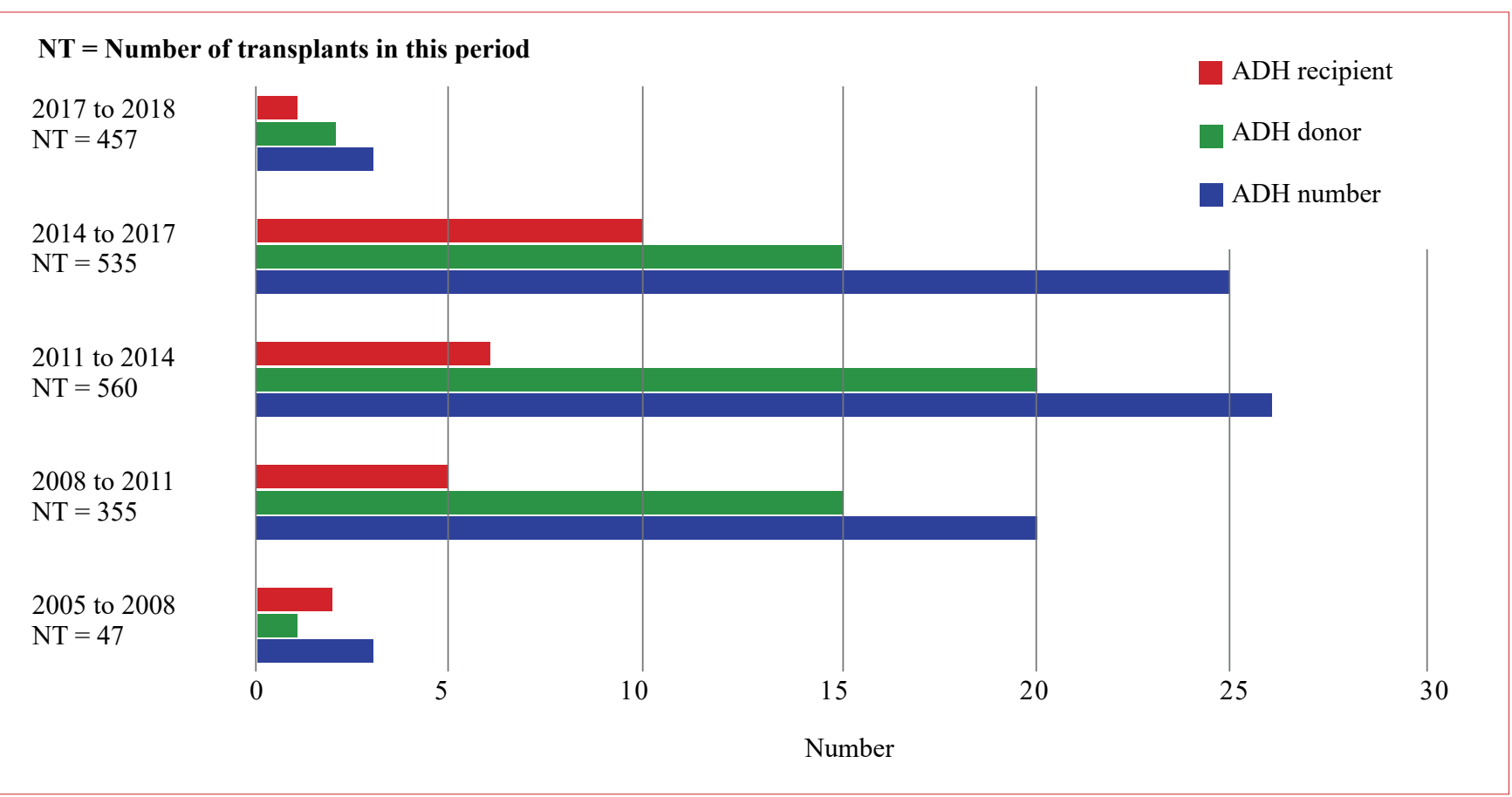

Figure 2: Distribution of ADH by years

(median: 25.9, mean \pm SD: $26.2 \pm 3.4$ ). Forty-one patients were male $(53.2 \%)$ whereas $36(46.8 \%)$ were female. The LDH was terminated due to various reasons at the following stages: cholecystectomy + cholangiography $(n=25)$, laparotomy + biopsy $(n=17)$, cholecystectomy + cholangiography + biopsy $(n=12)$, parenchymal transection $(n=8)$, laparoscopic biopsy $(n=6)$, laparotomy $(n=4)$, hilar dissection $(n=4)$ and parenchymal transection + division of left bile duct $(n=1)$. The distribution of ADH by years in relation to the total number of transplants per three-year period is shown in Figure 2.

\section{Donor reasons}

Fifty-three (68.8\%) LDH procedures were terminated due to donor related reasons.

\section{Unexpected liver parenchyma quality}

Thirty-two (41.5\%) LDH procedures were aborted due to histopathologically proven poor liver parenchyma quality. These included hepatosteatosis, fibrosis, chronic active hepatitis, gross granular parenchyma, cholestasis, and periportal inflammation. The most frequent histopathological problem in $\mathrm{ADH}$ was hepatosteatosis, whose degree varied from $5-40 \%(n=25)$ (Figure 3$)$. In one patient in which the procedure was aborted due to donor hepatosteatosis, re-evaluation after six months of diet and medical therapy showed resolution enabling the LDLT to be carried out as originally planned.

\section{Insufficient remnant liver volume not predictable by MDCT evaluation}

Four $(5.2 \%) \mathrm{LDH}$ procedures were terminated only due to the insufficient remnant liver volume. All four LLD candidates had borderline remnant liver volume (28-30\%) and after clamping the right hepatic artery and right branch of portal vein, the Cantlie's line formed near the falciform ligament which was more medial (to the left) than expected.
The procedures were aborted in order to avoid insufficient remnant volume.

\section{Unexpected biliary tree variations}

Five $(6.5 \%) \mathrm{LDH}$ procedures were terminated due to variations in the biliary tract. In addition, one of these 5 patients had concomitant insufficient liver volume and

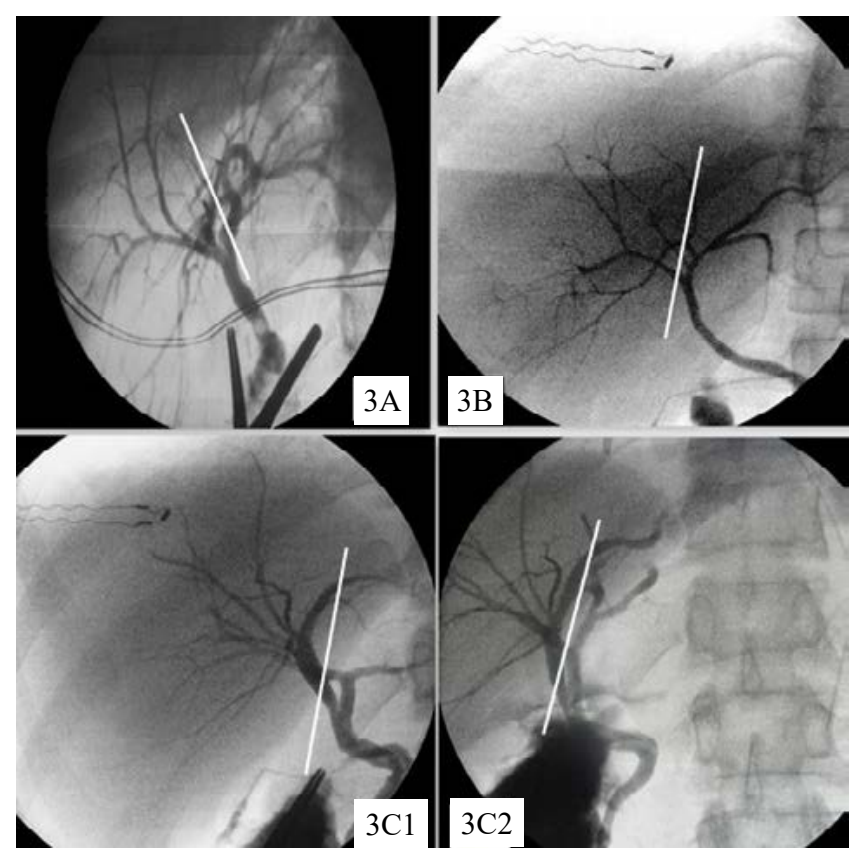

Figure 3: Predicted transection lines for right or left donor hepatectomy A) Intraoperative cholangiography of a left lobe donor hepatectomy; transection shows that after transection there are multiple transected bile ducts on both sides. B) Intraoperative cholangiogram of right lobe donor, transection line shows that there is a suspicion for injury to the segment 4 bile duct; this procedure could have proceeded with 3 bile duct stump. C1 and C2) Intraoperative cholangiography of the same patient from two different angles showing segment 2 bile duct draining into the right hepatic duct. 


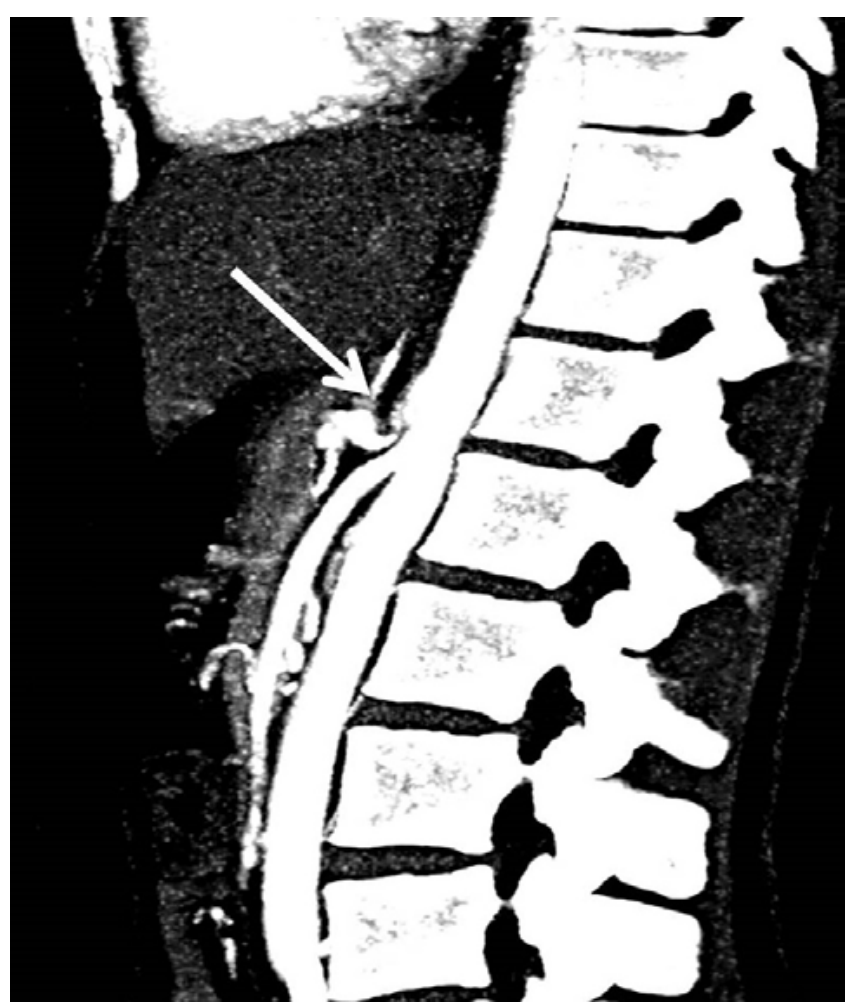

Figure 4: Sagittal section MDCT images showing median arcuate ligament syndrome (white arrow showing the compression of celiac axis)

another had hepatosteatosis. In one LLD in whom a left lateral segmentectomy was planned, intraoperative cholangiography revealed a connection between right and left hepatic bile ducts that precluded the intended partial hepatectomy (Figure 3A). Another LDH operation was aborted after intraoperative cholangiography showed that the segment 4 bile duct was at risk of injury. Normally LDH could have been performed that would include 3 bile duct stumps in the graft (Figure 3B). Another donor operation was aborted due to segment 2 biliary tract draining into right anterior sectoral bile duct (Figure 3C1-C2).

\section{Unexpected abnormal vascular structure}

Three $(3.9 \%) \mathrm{LDH}$ procedures were terminated due to vascular reasons. In one of these procedures hepatic arterial pulsation was weak during palpation of the hepatic hilum. Intraoperative Doppler ultrasonography showed weak flow in the arterial system that lacked systolic pulsation and the operation was aborted. Postoperatively meticulous re-evaluation of the MDCT revealed median arcuate ligament syndrome (MALS) (Figure 4). The other two operations were emergency LDLT procedures for acute liver failure when the senior surgeon was not present. In these procedures, after clamping the right hepatic artery and the portal vein, the Cantlie's line formed more medially at the left side than anticipated. The surgeon in charge of the operation terminated the operation due to insufficient graft volume. Re-evaluation of the donor dynamic liver

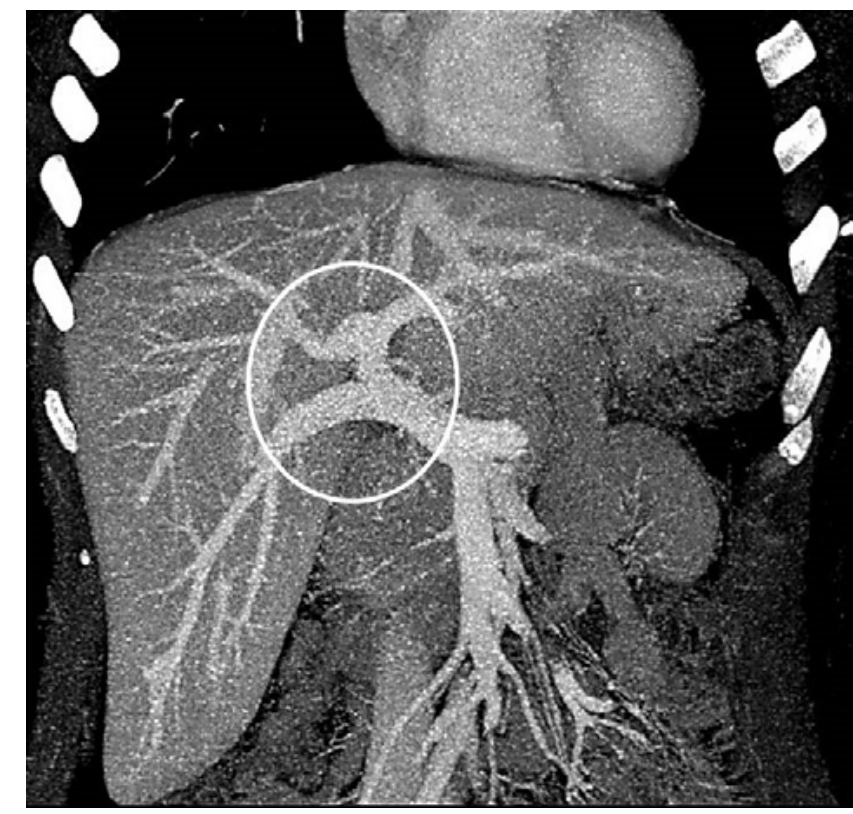

Figure 5: Coronal section MDCT images showing type 3 portal vein anatomy according to Varotti-Emre classification (White circle)

MDCT showed that the portal vein had a type 3 portal vein anatomical anomaly (Figure 5). These were the two cases in the beginning of the LDLT programme of our institute.

\section{Unexpected iatrogenic vascular injury}

Four $(5.2 \%) \mathrm{LDH}$ procedures were terminated due to iatrogenic surgical injury. In two LLDs, right hepatic arteries were dissected, and the intraoperative Doppler ultrasonography showed lack of blood flow in the right hepatic arteries so the procedures were aborted. In another procedure in which left lobectomy was planned, the segment 4 artery was accidently transected and therefore the operation was terminated. In the fourth case during the parenchyma transection it was seen that the middle hepatic vein was retained in the graft side in a case with a borderline remnant volume of $29 \%$ and the case was aborted due the insufficient remnant liver volume.

\section{Recipient reasons}

Twenty-four (48.3\%) LDH procedures were terminated due to recipient related reasons. Eleven procedures were

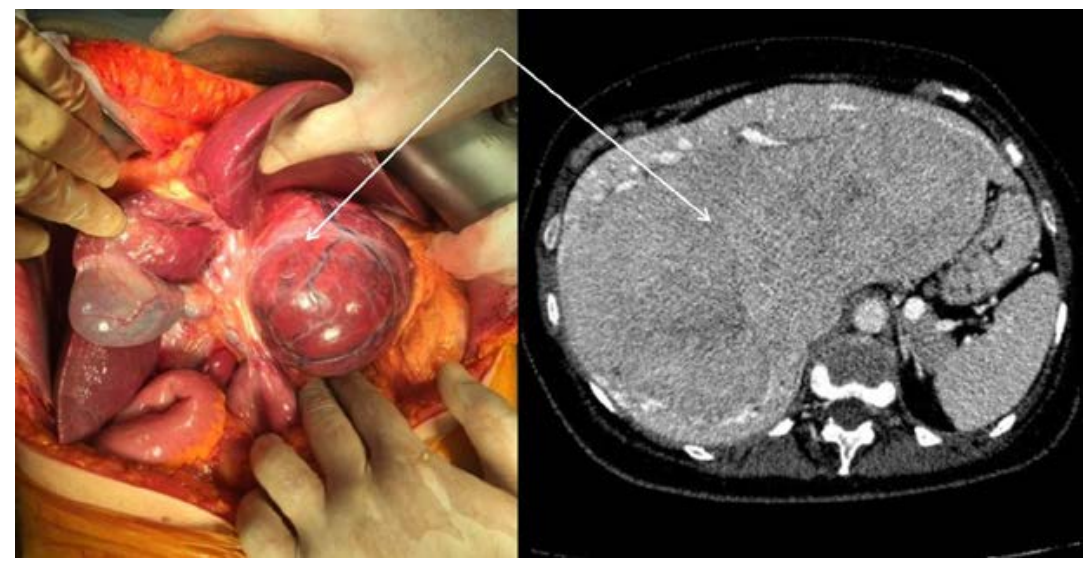

Figure 6: Intraoperative and preoperative MDCT images of the giant hepatic tumour (thin white arrows) 


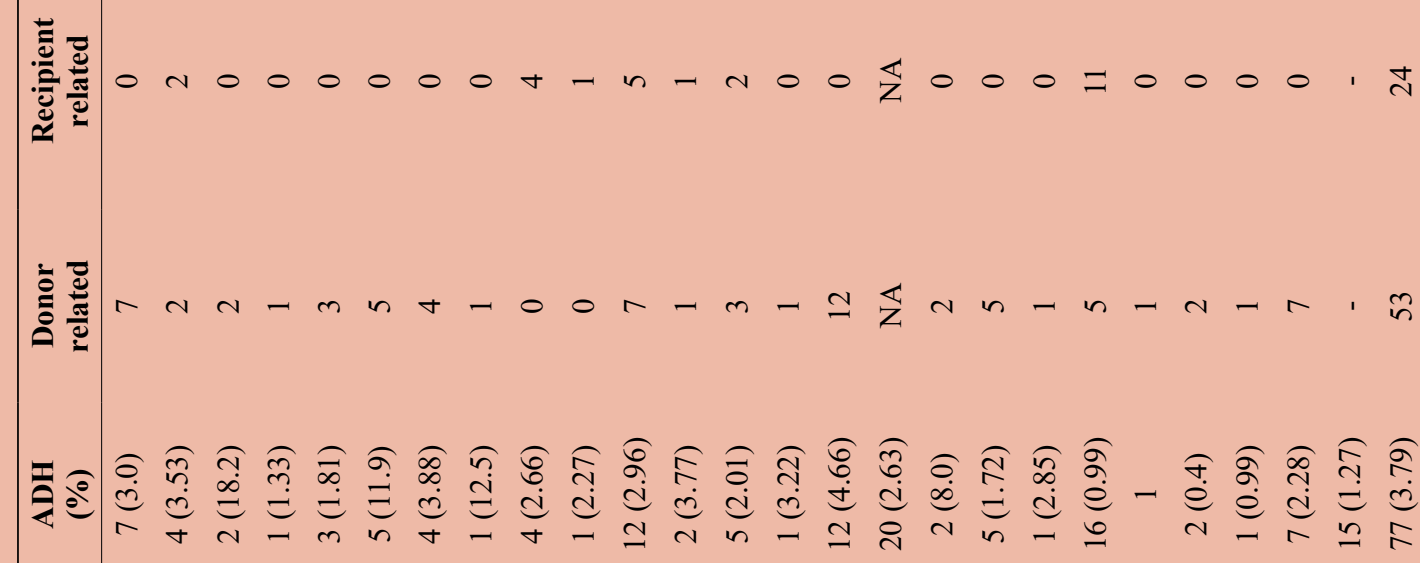

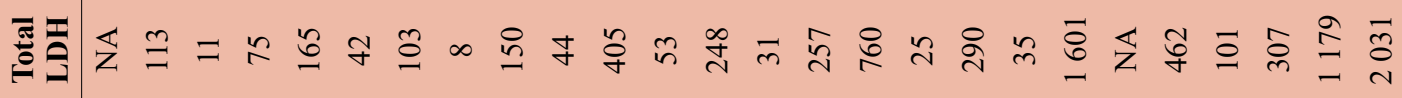

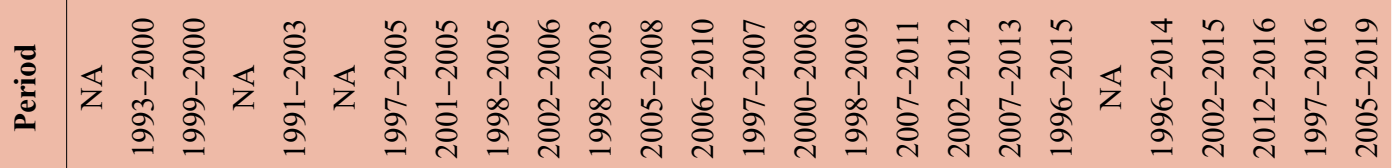

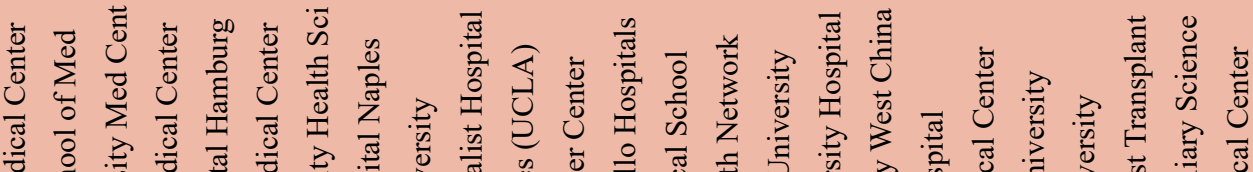

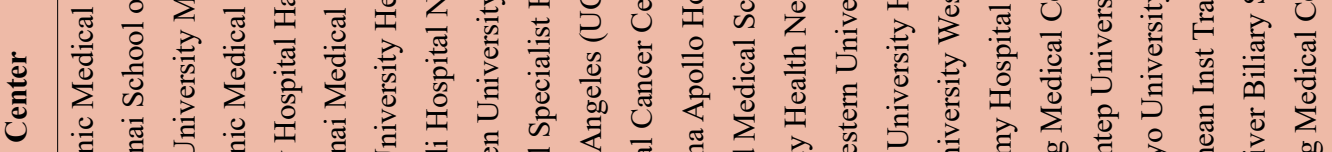

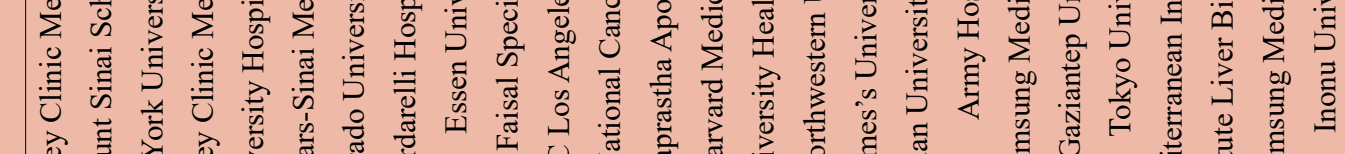

吾

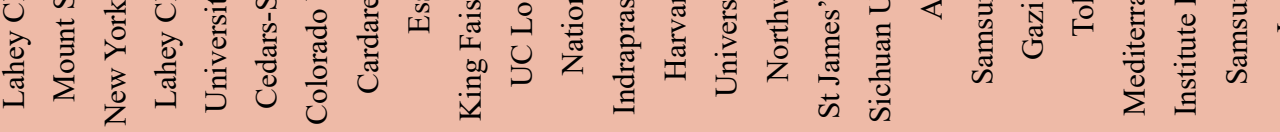

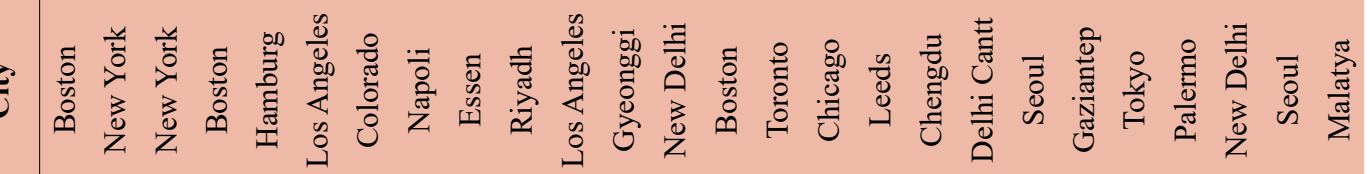

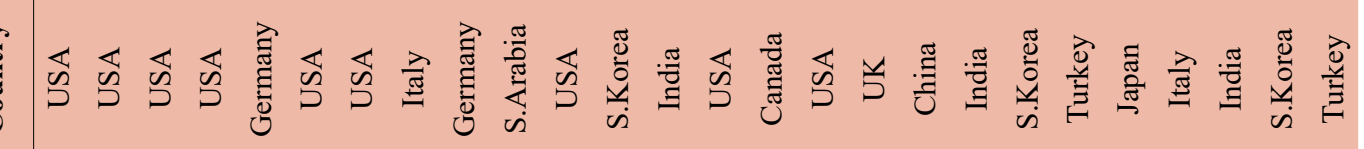

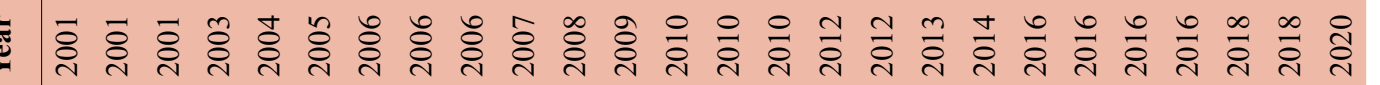


terminated because the recipient became haemodynamically unstable during the explant hepatectomy. Three LDH procedures were terminated because of excessive bleeding during dissection of the diffuse adhesions due to previous peritonitis. In four recipients, advanced stage tumour was detected, and the procedure was aborted. Another three recipients with refractory oesophagus variceal bleeding were scheduled for LT but they were found to have noncirrhotic portal hypertension confirmed on operative liver biopsy. They went on to have portocaval shunts and the LDH procedure was terminated. In another recipient with portal vein thrombosis, sufficient portal venous flow could not be obtained after thromboendovenectomy and the surgeon in charge of operation concluded that portal vein reconstruction was not possible. Therefore, both recipient and donor operations were terminated. Two patients with giant hepatic tumours were operated with the intent of LT. Intraoperatively, the remnant liver volumes were evaluated to be sufficient and extended right hepatectomies were performed and the LDH aborted (Figure 6).

\section{Clinical course of recipients}

In twenty-three of the 77 aborted procedures, the recipients underwent LDLT $(n=21)$ or DDLT $(n=2)$ in a median of 6 (range 0-129 days) days following the index procedure. In two patients whose procedure was aborted due to haemodynamic instability and another patient with hepatosteatosis, LDLT was performed at a later stage using the graft obtained from the index aborted donor candidate. Thirty-six cases did not have LT and died in a median of 17.5 (range 0-1 154 days) days following the aborted procedure. Eighteen patients are still under surveillance on the waiting list.

\section{Results of literature review}

The literature review analysis of the 25 published articles retrieved and the current series are summarised in Table 1 and Table 2..$^{5-8,10,11,17-35}$ Detailed information was obtained on 99 patients, $73(73.3 \%)$ ADHs were due to donor reasons while $26(26.7 \%)$ were due to reasons related to the recipient.
The most common donor related reasons were biliary tract variations $(27.4 \%)$, poor quality of parenchyma $(20.5 \%)$ and hepatosteatosis $(19.2 \%)$, while the most common recipient related reasons were haemodynamic problems (52.2\%).

\section{Discussion}

In this study, we aimed to describe our ADH experience as well as to review the studies published in the literature. According to our literature review, prevalence of $\mathrm{ADH}$ ranged from $0.4-18.2 \%{ }^{5-7,9,17-20,26}$ The prevalence of $\mathrm{ADH}$ in our institute was $3.79 \%$, which is consistent with the literature. Furthermore, $68.8 \%$ of the ADHs in our institute were due to donor related reasons and $31.2 \%$ of the ADHs were due to recipient related reasons. Our results showed that donor related $\mathrm{ADH}$ rates were lower than in the literature but recipient related $\mathrm{ADH}$ rates were higher. Although the overall ADH rates in our institute appear higher than many studies in the literature, our $\mathrm{ADH}$ rates have dramatically decreased since we started using preoperative MRI and MRCP. Today, unexpected quality of the donor liver parenchyma and the haemodynamic problems of the recipients are among the most common reasons for termination of LDH procedure in our transplant institute.

Hepatosteatosis was the main factor for graft parenchyma quality leading to ADHs. Debate regarding the efficacy of the radiologic instruments in determining the hepatosteatosis is still not resolved. The efficacy of MDCT in determining the hepatosteatosis increases with higher grades of hepatosteatosis. Sensitivity of MDCT in determining hepatosteatosis $\leq 30 \%$ ranges between $50-70 \%$. Sensitivity increases to $80 \%$ if hepatosteatosis is $>30 \%{ }^{36}$ In a study conducted in our institute, Sagir and colleagues ${ }^{37}$ stated that the sensitivity and specificity of MDCT in determining hepatosteatosis was reported to be $77 \%$ and $75 \%$ respectively. MRI has been reported to have more sensitivity in determining hepatosteatosis when compared to MDCT. However, MRI sensitivity also differs with different grades of hepatosteatosis and it ranges between $82-97.4 \% .{ }^{38}$ Although it is not yet included in the preoperative donor evaluation

\begin{tabular}{lcc} 
Table 2: Reasons for aborted donor hepatectomy in the reported series & References \\
\hline Reasons for aborted donor hepatectomy & Patients & 73 \\
\hline Donor related & 14 & $5,7,8,11,18,22-24,34$ \\
Hepatosteatosis/Steatohepatitis & 15 & $5-8,18,22,27$ \\
Poor quality of parenchyma/Fibrosis & 4 & $5,34,35$ \\
Hepatic artery variations & 3 & $18,26,31$ \\
Portal venous variations & 20 & $5-7,11,18,25-28,32,35$ \\
Biliary tract variations & 5 & $5,7,25,26,34$ \\
Insufficient remnant liver volume & 6 & $5,8,10,11,20,30$ \\
Hemodynamic instability/Bradicardia & 2 & 5,11 \\
Surgical conditions/Bleeding & 4 & $11,25,29,30$ \\
Miscellaneous & $\mathbf{2 6}$ & 22 \\
Recipients related & 1 & 22,23 \\
Dense adhesions & 6 & $6,22,25,27,33$ \\
Advanced stage tumor & 12 & $19,22,25,27$ \\
Hemodynamic instability/Mortality & 7 & 17 \\
Miscellaneous & $\mathbf{2 0}$ & \\
No-available information & &
\end{tabular}


criteria, transient elastography, which is frequently used to measure fibrosis and hepatosteatosis in chronic liver disease, can be used to evaluate donor parenchyma quality. ${ }^{39,40}$ In our early experience regarding the evaluation of LLD candidates, combined MRI and MDCT use was not frequently performed. For the last two years, we have started to routinely perform MRI combined with MDCT and termination due to hepatosteatosis was significantly decreased. Unfortunately, MRI cannot be performed for LLD evaluation in cases that require emergency LT which requires rapid evaluation and decision for operation, and therefore we cannot use MRI for evaluation of parenchymal quality of LLDs under extreme emergency conditions. In recipients that require emergency LT, LLD candidates with low BMI and no hepatosteatosis on MDCT are chosen. In these cases, laparoscopic or open biopsy is obtained when necessary and, if the procedure is aborted due to unexpected parenchymal problem, no significant morbidity is observed. In these cases, if the perioperative cholangiography shows multiple biliary ducts, this creates a potential postoperative complication risk for the recipient but not for the LLDs. A detailed informed consent is obtained regarding the possible outcomes. Abnormal bile duct anatomy is very rarely a reason for $\mathrm{ADH}$ due to potential risks for the LLDs.

Another important issue is the relationship between BMI and hepatosteatosis in LLD candidates. Jehangir and colleagues ${ }^{41}$ claimed that there was no significant correlation between BMI and hepatosteatosis $(r=0.13)$, however, Rinella and colleagues ${ }^{42}$ showed a significant correlation $(r=0.49)$ between BMI and hepatosteatosis in their study. When we evaluated the correlation analysis of both studies, it was understood that there was no moderate or high relationship between BMI and hepatosteatosis. Twenty-one of the LLDs presented in this study were aborted due to hepatosteatosis $(\geq 10 \%)$ and their mean \pm SD BMI was $28.1 \pm 4.0 \mathrm{~kg} / \mathrm{m}^{2}$. Even if the hepatosteatosis is below 5\% in CT and MRI, we routinely perform liver biopsy at the beginning of surgery in LLDs with $B M I \geq 28 \mathrm{~kg} / \mathrm{m}^{2}$.

Perkins and colleagues ${ }^{43}$ reported that some LLD candidates who are not considered eligible for donation due to hepatosteatosis can be re-evaluated after an appropriate diet and medical treatment and can become suitable donors. In our study, one LLD candidate whose operation had been terminated due to $40 \%$ hepatosteatosis on frozen biopsy was re-evaluated after a six-months of diet and medical therapy and underwent an uneventful $\mathrm{LDH}$ with an uneventful postoperative recovery. It should be kept in mind that if the recipient has no other LLD candidate, any candidate with hepatosteatosis can become eligible for LDLT after a period of diet and medical therapy.

The bile duct variations can be preoperatively evaluated by MRCP, which is effective in showing the normal biliary duct anatomy, but its success in demonstrating the variations of the bile ducts is low. Lee and collegues ${ }^{44}$ showed that intraoperative cholangiography and MRCP coincided in $94 \%$ of the cases and MRCP predictions were erroneous in $6 \%$ of the cases. They also showed that sensitivity of MRCP was $70 \%$. The choice of evaluation of the donor bile ducts with MRCP changes according to transplantation centers. ${ }^{45}$ Despite the normal appearance of the bile ducts in preoperative MRCP, we routinely perform intraoperative cholangiography.
Insufficiency of remnant liver volume is characterised by postoperative prolonged cholestasis, coagulopathy and ascites. ${ }^{46} \mathrm{In}$ order to avoid this problem in LLDs, we think that $\geq 35 \%$ remnant volume in donor candidates over 35 years and $\geq 30 \%$ remnant volume in donor candidates under 35 years old should be anticipated. The ultimate decision to continue resection of the right lobe should be given after transient clamping the right portal vein and hepatic artery branches and observing the Cantlie's line.

Preoperative MDCT is effective in revealing the vascular anatomy of the liver. In the present study, the detection of a weak hepatic artery pulsation in the hepatoduodenal ligament exploration was the cause of the termination in a patient. Retrospective evaluation of the patient's MDCT revealed a MALS. MALS prevalence is reported to be $2-24 \%$ and it is not a contraindication for $\mathrm{LDH}^{4}{ }^{47}$ In asymptomatic MALS cases in which arterial pulsation cannot be obtained with palpation or Doppler ultrasonography, it can be thought that an adequate arterial flow can be provided with various surgical techniques. However, the necessity for treatment of asymptomatic MALS in LLD candidates who have no adequate arterial flow in the hepatic artery should be assessed thoroughly. The main reason of termination of the LDH is due to the fact that both our experience and the information in literature were inconclusive regarding the impact of weak pulsation in the left branch of the hepatic artery in supplying the remnant liver of the donor.

Only "dragon tail-type" portal vein anatomy is an absolute contraindication for $\mathrm{LDH}^{48}$ In our study, type 3 portal vein anatomy was the cause of terminating the two ADH procedures. Type 3 portal vein anatomy is not a contraindication for LDLT. However, these cases were in the beginning of our transplant programme. Many technical modifications in reconstruction of dual portal vein are defined. ${ }^{49}$ Currently, we use the technique that we have developed and defined as "Malatya Approach" to reconstruct portal vein anastomosis in right lobe LDLT. ${ }^{50}$ Four cases that were terminated due to surgical reasons happened in the beginning of the learning curve of the donor surgeons. Together with the completion of the learning curve, which is calculated as $200 \mathrm{LDHs}$ for transplant centres, ADH due to iatrogenic biliovascular injury was reduced below the acceptable limits. ${ }^{4,50}$

In order to reduce $\mathrm{ADHs}$, recipients should also be evaluated thoroughly. Cheah and colleagues ${ }^{9}$ reported that $25.9 \%$ of ADHs were related to recipient and the most common recipient related reasons was due to haemodynamic instability and encountering advanced malignancy during laparotomy. We consider that initiating the recipient procedure to assess the resectability of the tumour comprehensively before the donor operation will reduce the unnecessary ADHs in cases of giant liver tumours without cirrhosis. Similarly, in cases suspected to have an advanced malignancy, recipient procedure should precede the donor procedure in order to provide detailed examination of the peritoneal spread of the tumour. We believe this approach will substantially decrease the unnecessary ADHs. It should be kept in mind that dense and fibrotic intraoperative adhesions would cause difficulty in dissection and massive bleeding that would preclude the recipient and donor operation. In such cases, it would be suitable to start the recipient procedure before the donor procedure. In case of portal vein thrombosis, LDH should proceed after assessing 
the availability of obtaining sufficient portal venous flow for the recipient.

Retrospective evaluation of our $\mathrm{ADH}$ results showed that some of the donor and recipient related terminations could have been reduced with performing appropriate intraoperative measures. In summary, ADHs due to MALS and anomalous portal venous branching could have been avoided by a meticulous radiological evaluation prior to surgery. If liver parenchyma quality of about $40 \%$ of our aborted donors had been evaluated by preoperative USguided biopsy, most of the donors would either have been eliminated directly or re-evaluated after the intensive diet program. In fact, although this seems as the most ideal strategy, the majority of our patients do not accept this approach. However, sometimes the intraoperative macroscopic appearance can give valuable information as much as the biopsy; even though the pathology result is normal, we have aborted a few procedures according to the macroscopic appearance. Recipient related reasons such as adhesions $(n=3)$, advanced tumours $(n=4)$, PVT $(n=1)$ and resectable tumours $(n=2)$ could have been prevented by proceeding with the recipient procedure before the donor surgery.

\section{Conclusions}

Despite all public awareness campaigns in organ donation, LDLT still remains the only option for patients with chronic liver disease in Asia and the Middle East countries. In order to reduce the rate of $\mathrm{ADH}$, donor candidates should be evaluated according to the preoperative donor evaluation algorithm. These algorithms are based on the experiences of LDLT high-volume centres. At our institute, donor age, BMI and remnant liver volume $>30 \%$ are essential in our decision to proceed with donor operation in order to prevent ADH. We routinely perform MRCP to delineate donor biliary tree anatomy in the preoperative period. In prospective donors with hepatosteatosis, we perform either a preoperative percutaneous, a mini-laparotomy or laparoscopic liver biopsy before proceeding to the donor operation. If the recipient's condition is critical, we start the recipient operation before the donor operation, to ensure liver explantation is feasible before committing to a partial hepatectomy on the donor. In LDLT, the safety of the LLD has paramount importance; if donor safety is in doubt, surgeons should not hesitate to terminate the operation in order to prevent mortality and morbidity.

\section{Conflict of interest}

The authors declare no conflict of interest.

\section{Funding source}

The authors received no financial support for this study.

\section{Ethical approval}

This study was prepared after ethical approval from the Inonu University Rectorate Ethics Committee (Approval No: 2019/4-46). The study was also conducted in accordance with the guidelines of the Declaration of Helsinki.

\section{ORCID}

K Kutluturk (D) https://orcid.org/0000-0002-7030-4953

S Akbulut (D) https://orcid.org/0000-0002-6864-7711

A Baskiran (D) https://orcid.org/0000-0002-7536-1631
F Gonultas (i) https://orcid.org/0000-0001-7771-3891

A Dirican (I) https://orcid.org/0000-0002-8647-3268

B Isik (D) https://orcid.org/0000-0002-2395-3985

S Yilmaz (iD) https://orcid.org/0000-0002-8044-0297

\section{REFERENCES}

1. Koc C, Akbulut S, Ozdemir F, et al. Analysis of risk factors affecting the development of infection in artificial vascular grafts used for reconstruction of middle hepatic vein tributaries in living donor liver transplantation. Transplantation. 2019 Feb 4. PMID: 30747841. https://doi.org/10.1097/ TP.0000000000002583.

2. Akbulut S, Yilmaz S. Liver transplantation in Turkey: historical review and future perspectives. Transplant Rev (Orlando). 2015;29(3):161-7. PMID: 25535023. https://doi. org/10.1016/j.trre.2014.12.002.

3. Arshad A, Anderson B, Sharif A. Comparison of organ donation and transplantation rates between opt-out and opt-in systems. Kidney Int. 2019;95(6):1453-60. PMID: 31010718. https://doi.org/10.1016/j.kint.2019.01.036.

4. Onur A, Akbulut S, Dirican A, Isik B, Yilmaz S. Lifethreatening or nearly life-threatening complications in living liver donors. Clin Transplant. 2018;32(6):e13262. PMID: 29665076. https://doi.org/10.1111/ctr.13262.

5. Guba M, Adcock L, MacLeod C, et al. Intraoperative 'no go' donor hepatectomies in living donor liver transplantation. Am J Transplant. 2010;10(3):612-8. PMID: 20121746. https://doi. org/10.1111/j.1600-6143.2009.02979.x.

6. Vij V, Ramaswamy VK, Goja S, et al. Intraoperative no go donor hepatectomy in living donor liver transplantation. Am J Transplant. 2010;10(9):2181-2. PMID: 20883553. https://doi. $\operatorname{org} / 10.1111 / \mathrm{j} .1600-6143.2010 .03155 . x$.

7. Lei JY, Yan LN. Intraoperative "no go" donor hepatectomy in living donor liver transplantation. Transplant Proc. 2013;45(6):2253-7. PMID: 23953536. https://doi. org/10.1016/j.transproceed.2013.02.119.

8. Pamecha V, Bharathy KGS, Mahansaria SS, et al. "No go" donor hepatectomy in living-donor liver transplantation. Hepatol Int. 2018;12(1):67-74. PMID: 29170994. https://doi. org/10.1007/s12072-017-9832-z.

9. Cheah YL, Simpson MA, Pomposelli JJ, Pomfret EA. Incidence of death and potentially life-threatening near-miss events in living donor hepatic lobectomy: a world-wide survey. Liver Transpl. 2013;19(5):499-506. PMID: 23172840. https://doi.org/10.1002/lt.23575.

10. Pomfret EA. Early and late complications in the right-lobe adult living donor. Liver Transpl. 2003;9(10 Suppl 2):S45-9. PMID: 14528428. https://doi.org/10.1053/jlts.2003.50231.

11. Pomfret EA, Pomposelli JJ, Jenkins RL. Live donor liver transplantation. J Hepatol. 2001;34(4):613-24. PMID: 11394665. https://doi.org/10.1016/S0168-8278(01)00031-9.

12. Dirican A, Baskiran A, Dogan M, et al. Evaluation of potential liver donor in living donor liver transplantation. Transplant Proc. 2015;47(5):1315-8. PMID: 26093708. https://doi. org/10.1016/j.transproceed.2015.04.045.

13. Lee SD, Kim SH, Kim YK, Lee SA, Park SJ. Graft-to-recipient weight ratio lower to $0.7 \%$ is safe without portal pressure modulation in right-lobe living donor liver transplantation with favorable conditions. Hepatobiliary Pancreat Dis Int. 2014;13(1):18-24. PMID: 24463075. https://doi.org/10.1016/ S1499-3872(14)60002-3.

14. Uemura T, Wada S, Kaido T, et al. How far can we lower graft-to-recipient weight ratio for living donor liver 
transplantation under modulation of portal venous pressure? Surgery. 2016;159(6):1623-30. PMID: 26936527. https://doi. org/10.1016/j.surg.2016.01.009.

15. Soin AS, Chaudhary RJ, Pahari H, Pomfret EA. A worldwide survey of live liver donor selection policies at 24 centres with a combined experience of 19009 adult living donor liver transplants. Transplantation. 2019;103(2):e39-e47. PMID: 30308575. https://doi.org/10.1097/TP.0000000000002475.

16. Lee SG. A complete treatment of adult living donor liver transplantation: a review of surgical technique and current challenges to expand indication of patients. Am J Transplant. 2015;15(1):17-38. PMID: 25358749. https://doi.org/10.1111/ ajt.12907.

17. Abecassis MM, Fisher RA, Olthoff KM, et al. Complications of living donor hepatic lobectomy - a comprehensive report. Am J Transplant. 2012;12(5):1208-17. PMID: 22335782. https://doi.org/10.1111/j.1600-6143.2011.03972.x.

18. Trotter JF, Campsen J, Bak T, et al. Outcomes of donor evaluations for adult-to-adult right hepatic lobe living donor liver transplantation. Am J Transplant. 2006;6(8):1882-9. PMID: 16889543. https://doi.org/10.1111/j.1600-6143.2006. 01322.x.

19. Khalaf H, Al-Sofayan M, El-Sheikh Y, et al. Donor outcome after living liver donation: a single-centre experience. Transplant Proc. 2007;39(4):829-34. PMID: 17524825. https://doi.org/10.1016/j.transproceed.2007.03.003.

20. Rao PP, Routh D, Naidu CS, et al. Donor outcome in live-related liver transplantation. Med J Armed Forces India. 2014;70(2):100-4. PMID: 24843195. https://doi. org/10.1016/j.mjafi.2013.10.016.

21. Park J, Choi GS, Gwak MS, et al. Intraoperative abortion of adult living donor liver transplantation: 15 cases from 1,179 cases in 20 years of experience in a single centre. Ann Surg Treat Res. 2018;95(4):213-221. PMID: 30310804. https://doi. org/10.4174/astr.2018.95.4.213.

22. Lee J, Choi G, Cho W, et al. Review of aborted hepatectomy of liver transplantation in a single centre study. Am J Transplant. 2016;16 (Suppl 3):5-798. https://doi.org/10.1111/ajt.13895.

23. Kim SH, Cho SY, Park SJ, et al. Learning curve for livingdonor liver transplantation in a fledgling cancer centre. Transpl Int. 2009;22(12):1164-71. PMID: 19891045. https:// doi.org/10.1111/j.1432-2277.2009. 00934.x.

24. Saidi RF, Elias N, Ko DS, et al. Live donor partial hepatectomy for liver transplantation: is there a learning curve? Int J Organ Transplant Med. 2010;1(3):125-30. PMID: 25013578.

25. Ghobrial RM, Freise CE, Trotter JF, et al. Donor morbidity after living donation for liver transplantation. Gastroenterology. 2008;135(2):468-76. PMID: 18505689. https://doi.org/10.1053/j.gastro.2008.04.018.

26. Shackleton CR, Vierling JM, Nissen N, et al. Morbidity in live liver donors: standards-based adverse event reporting further refined. Arch Surg. 2005;140(9):888-95. PMID: 16172298. https://doi.org/10.1001/archsurg.140.9.888.

27. Miller CM, Gondolesi GE, Florman S, et al. One hundred nine living donor liver transplants in adults and children: a singlecentre experience. Ann Surg. 2001;234(3):301-11. PMID: 1689992.

28. Lee VS, Morgan GR, Teperman LW, et al. MR imaging as the sole preoperative imaging modality for right hepatectomy: a prospective study of living adult-to-adult liver donor candidates. AJR Am J Roentgenol. 2001;176(6):1475-82. PMID: 11373217. https://doi.org/10.2214/ajr.176.6.1761475.
29. Yildiz F, Coban S. A rare unsuspected condition to abandon liver donation operation, Peliosis Hepatis: A case report. HPB 2016;18(S1):e385-601. https://doi.org/10.1016/j. hpb.2016.03.550.

30. Akamatsu N, Kokudo N. Living liver donor selection and resection at the University of Tokyo Hospital. Transplant Proc. 2016;48(4):998-1002. PMID: 27320541. https://doi. org/10.1016/j.transproceed.2016.01.019.

31. Gruttadauria S, Pagano D, Petridis I, et al. Complications and near-miss events after hepatectomy for living-related liver donation: an Italian single centre report of one hundred cases. Ann Transplant. 2016; 21:596-601. PMID: 27653736. https:// doi.org/10.12659/aot.899662.

32. Cuomo O, Ragozzino A, Iovine L, et al. Living donor liver transplantation: early single-centre experience. Transplant Proc. 2006;38(4):1101-5. PMID: 16757277. https://doi. org/10.1016/j.transproceed.2006.02.150.

33. Nadalin S, Malagó M, Testa G, et al. "Hepar divisum"-as a rare donor complication after intraoperative mortality of the recipient of an intended living donor liver transplantation. Liver Transpl. 2006;12(3):428-34. PMID: 16498667. https:// doi.org/10.1002/lt.20723.

34. Broering DC, Wilms C, Bok P, et al. Evolution of donor morbidity in living related liver transplantation: a singlecentre analysis of 165 cases. Ann Surg. 2004;240(6):101324. PMID: 15570207. https://doi.org/10.1097/01. sla.0000146146.97485.6c.

35. Hakeem A, Raza S, Reddy M, et al. Donor morbidity following living donor liver transplantation (LDLT): outcome from a small volume centre. Gut 2012;61(Suppl 2). https:// doi.org/10.1136/gutjnl-2012-302514c.51.

36. Ryan CK, Johnson LA, Germin BI, Marcos A. One hundred consecutive hepatic biopsies in the workup of living donors for right lobe liver transplantation. Liver Transpl. 2002;8(12):1114-22. PMID: 12474149. https://doi. $\operatorname{org} / 10.1053 / \mathrm{jlts} .2002 .36740$.

37. Sagir KA, Karakas HM, Kirimlioglu H, et al. The assessment of hepatosteatosis in living-donor liver transplant: comparison of liver attenuation index and histopathologic results. Exp Clin Transplant. 2017;15(1):69-77. PMID: 26221870. https:// doi.org/10.6002/ect.2015.0043.

38. Bohte AE, Van Werven JR, Bipat S, Stoker J. The diagnostic accuracy of US, CT, MRI and 1H-MRS for the evaluation of hepatic steatosis compared with liver biopsy: a meta-analysis. Eur Radiol. 2011;21(1):87-97. https://doi.org/10.1007/ s00330-010-1905-5.

39. Yen YH, Kuo FY, Lin CC, et al. Predicting hepatic steatosis in living liver donors via controlled attenuation parameter. Transplant Proc. 2018;50(10):3533-38. PMID: 30577232. https://doi.org/10.1016/j.transproceed.2018.06.039.

40. Kim JM, Ha SY, Joh JW, et al. Predicting hepatic steatosis in living liver donors via noninvasive methods. Medicine (Baltimore). 2016;95(7):e2718. PMID: 26886612. https://doi. org/10.1097/MD.0000000000002718.

41. Jehangir M, Nazir R, Jang A, et al. Macrovesicular steatosis in living related liver donors: correlation of biopsy findings with CT liver attenuation index and body mass index. Clin Transplant. 2016;30(9):1016-20. PMID: 27291347. https:// doi.org/10.1111/ctr.12782.

42. Rinella ME, Alonso E, Rao S, et al. Body mass index as a predictor of hepatic steatosis in living liver donors. Liver Transpl. 2001;7(5):409-14. PMID: 11349260. https://doi. org/10.1053/jlts.2001.23787. 
43. Perkins JD. Saying 'Yes' to obese living liver donors: short-term intensive treatment for donors with hepatic steatosis in livingdonor liver transplantation. Liver Transpl. 2006;12(6):1012-3. PMID: 16721781. https://doi.org/10.1002/lt.20807.

44. Lee VS, Krinsky GA, Nazzaro CA, et al. Defining intrahepatic biliary anatomy in living liver transplant donor candidates at mangafodipir trisodium-enhanced MR cholangiography versus conventional T2-weighted MR cholangiography. Radiology. 2004;233(3):659-66. PMID: 15516606. https://doi.org/10.1148/radiol.2333031977.

45. Brown RS Jr, Russo MW, Lai M, et al. A survey of liver transplantation from living adult donors in the United States. N Engl J Med. 2003;348(9):818-25. PMID: 12606737. https:// doi.org/10.1056/NEJMsa021345.

46. Tucker ON, Heaton N. The 'small for size' liver syndrome. Curr Opin Crit Care. 2005;11(2):150-5. PMID: 15758596. https:// doi.org/10.1097/01.ccx.0000157080.11117.45.
47. Akamatsu N, Sugawara Y, Tamura S, et al Impact of celiac axis stenosis on living donor hepatectomy. Transplant Proc. 2006;38(9):2948-50. PMID: 17112871. https://doi. org/10.1016/j.transproceed.2006.08.107.

48. Duran C, Taner B, Dayangac M, et al. Why we did not use this donor. Liver Transpl. 2007;13(8):1199-200. PMID: 17663396. https://doi.org/10.1002/lt.21140.

49. Nakamura T, Tanaka K, Kiuchi T, et al. Anatomical variations and surgical strategies in right lobe living donor liver transplantation: lessons from 120 cases. Transplantation. 2002;73(12):1896-903. PMID: 12131684. https://doi org/10.1097/00007890-200206270-00008.

50. Yilmaz S, Kayaalp C, Isik B, et al. Reconstruction of anomalous portal venous branching in right lobe living donor liver transplantation: Malatya approach. Liver Transpl. 2017;23(6):751-761. PMID: 28240812. https://doi. org/10.1002/1t.24753. 